Pacific Journal of Mathematics

NON-HAUSDORFF MULTIFUNCTION GENERALIZATION OF 


\title{
NON-HAUSDORFF MULTIFUNCTION GENERALIZATION OF THE KELLEY-MORSE ASCOLI THEOREM
}

\author{
Geoffrey Fox And Pedro Morales
}

\begin{abstract}
The paper generalizes the Kelley-Morse theorem to continuous point-compact multifunction context. The generalization, which is non-Hausdorff, contains the Ascoli theorem for continuous functions on a $k_{3}$-space by the authors and the known multifunction Ascoli theorems of Mancuso and of Smithson.
\end{abstract}

1. Introduction. The Kelley-Morse theorem [3, p. 236] is central among the topological Ascoli theorems for continuous functions on a $k$-space. It generalizes to the $k_{3}$-space theorem of [1], which contains all known Ascoli theorems for $k$-spaces or $k_{3}$-spaces.

Obviously a multifunction generalization depends on a multifunction extension of "even continuity". One such extension is that of Lin and Rose [5], but this was not applied in Kelley-Morse context. Another which was so applied [7, p. 24] is two-fold and leads to a two-fold multifunction Kelley-Morse theorem which, however, does not contain the Mancuso theorem [6, p. 470], nor the Smithson theorem [9, p. 259]. This paper gives a natural multifunction extension of the definition and leads to a multifunction theorem containing all the above-mentioned theorems.

2. Tychonoff sets. Let $X$ and $Y$ be nonempty sets. A multifunction is a point to set correspondence $f: X \rightarrow Y$ such that, for all $x \in X, f x$ is a nonempty subset of $Y$. For $A \subseteq X, B \subseteq Y$ it is customary to write $f(A)=\bigcup_{x \in A} f x, f^{-}(B)=\{x: x \in X$ and $f x \cap B \neq \varnothing\}$ and $f^{+}(B)=$ $\{x: x \in X$ and $f x \subseteq B\}$. If $Y$ is a topological space, a multifunction $f: X \rightarrow Y$ is point-compact if $f x$ is compact for all $x \in X$.

Let $\left\{Y_{x}\right\}_{x \in X}$ be a family of nonempty sets. The $m$-product $P\left\{Y_{x}: x \in\right.$ $X$ \} of the $Y_{x}$ is the set of all multifunctions $f: X \rightarrow \cup_{x \in X} Y_{x}$ such that $f x \subseteq Y_{x}$ for all $x \in X$. In the case $Y_{x}=Y$ for all $x \in X$, the $m$-product of the $Y_{x}$, denoted $Y^{m X}$, is the set of all multifunctions on $X$ to $Y$. In particular, if $Y$ is a topological space, the symbol $\left(Y^{m X}\right)_{0}$ will denote the set of all point-compact members of $Y^{m X}$. For $x \in X$, the $x$-projection $\operatorname{pr}_{x}: P\left\{Y_{x}: x \in X\right\} \rightarrow Y_{x}$ is the multifunction defined by $\operatorname{pr}_{x} f=f x$. If the $Y_{x}$ are topological spaces, the pointwise topology $\tau_{p}$ on $P\left\{Y_{x}: x \in X\right\}$ is defined to be the topology having as open subbase the sets of the forms $\operatorname{pr}_{x}^{-}\left(U_{x}\right), \operatorname{pr}_{x}^{+}\left(U_{x}\right)$, where $U_{x}$ is open in $Y_{x}, x \in X$.

For $F \subseteq Y^{m X}, x \in X$, we write $F[x]=\bigcup_{f \in F} f x$. Let $Y$ be a topological space. A subset $F$ of $Y^{m X}$ is pointwise bounded if $F[x]$ has compact 
closure in $Y$ for all $x \in X$. A subset $T$ of $Y^{m x}$ is Tychonoff if, for every pointwise bounded subset $F$ of $T, T \cap P\{\overline{F[x]:} x \in X\}$ is $\tau_{p}$-compact. The following sets are Tychonoff:

(1) $Y^{X}$, by the classical Tychonoff theorem.

(2) $Y^{m X}$, by the theorem of Lin [4, p. 400].

(3) The set of all point-closed members of $Y^{m x}$, by Corollary 7.5 of [7, p. 17].

(4) $\left(Y^{m X}\right)_{0}$, by Corollary 7.6 of $[7$, p. 17].

LEMMA 2.1. If $F$ is a pointwise bounded subset of a Tychonoff set $T$, then the $\tau_{p}$-closure of $F$ in $T$ is compact.

Proof. Let $\bar{F}$ denote the $\tau_{p}$-closure of $F$ in $T$. Since $T \cap$

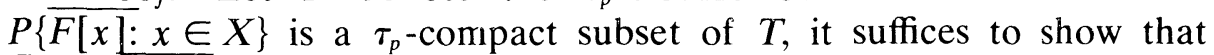
$\bar{F} \subseteq P\{F[x]: x \in X\}$. But this follows from Lemma 7.7 of [7, p. 17].

3. Even continuity. Let $X$ and $Y$ be topological spaces. A multifunction $f: X \rightarrow Y$ is lower semi-continuous (upper semicontinuous ) if $f^{-}(U)\left(f^{+}(U)\right)$ is open in $X$ whenever $U$ is open in $Y$. If $f$ is both lower semi-continuous and upper serni-continuous it is called continuous. Henceforth, the set of all continuous multifunctions on $X$ to $Y$ will be denoted $\mathscr{C}(X, Y)$. The multifunction $(f, x) \rightarrow f x$ on $Y^{m X} \times X$ to $Y$, or any restriction, will be denoted by the symbol $\omega$. Let $F \subseteq Y^{m X}$. A topology $\tau$ on $F$ is said to be jointly continuous if $\omega:(F, \tau) \times X \rightarrow Y$ is continuous.

A subset $F$ of $Y^{m x}$ is evenly contrnuous if, whenever $x \in X, K$ is a compact subset of $Y$ and $V$ is a neighborhood of $K$, there exist neighborhoods $U, W$ of $x, K$, respectively, such that

(a) $f \in F$ and $f x \cap W \neq \varnothing$ imply $U \subseteq f^{-}(V)$, and

(b) $f \in F$ and $f x \subseteq W$ imply $U \subseteq f^{+}(V)$.

This extends the original Kelley-Morse definition [3, p. 235] by the substitution of compact subsets of $Y$ for points of $Y$. It is easily verified that every member of an evenly continuous subset of $Y^{m X}$ is lower semi-continuous. Moreover, every member of an evenly continuous subset of $\left(Y^{m X}\right)_{0}$ is also upper semi-continuous, hence continuous.

LEMMA 3.1. Let $Y$ be a regular space. If $F$ is an evenly continuous subset of $Y^{m x}$, then the $\tau_{p}$-closure of $F$ in $Y^{m X}$ is evenly continuous.

Proof. Let $\bar{F}$ denote the $\tau_{p}$-closure of $F$ in $Y^{m x}$. Let $x \in X$, let $K$ be a compact subset of $Y$ and let $V$ be a closed neighborhood of $K$. There exist open neighborhoods $U, W$ of $x, K$, respectively, such that, for all $f \in F, f x \cap W \neq \varnothing$ implies $U \subseteq f^{-}(V)$ and $f x \subseteq W$ implies $U \subseteq f^{+}(V)$. Let $g \in \bar{F}$ be such that $g x \cap W \neq \varnothing$. Let $\left\{g_{\alpha}\right\}$ be a net in $F$ 
which is $\tau_{p}$-convergent to $g$. Since $\left\{h: h \in Y^{m x}\right.$ and $\left.h x \cap W \neq \varnothing\right\}$ is a $\tau_{p}$-neighborhood of $g, g_{\alpha} x \cap W \neq \varnothing$ eventually, so $U \subseteq g_{\alpha}^{-}(V)$ eventually. Suppose that $U \not \subset g^{-}(V)$. Then, for some $u \in U, g u \subseteq Y-V$, so $g_{\alpha} u \subseteq Y-V$ eventually, which is a contradiction.

Now let $g \in \vec{F}$ be such that $g x \subseteq W$. Let $\left\{g_{\alpha}\right\}$ be a net in $F$ which is $\tau_{p}$-convergent to $g$. Since $\left\{h: h \in Y^{m x}\right.$ and $\left.h x \subseteq W\right\}$ is a $\tau_{p}$ neighborhood of $g, g_{\alpha} x \subseteq W$ eventually, so $U \subseteq g_{\alpha}^{+}(V)$ eventually. Suppose that $U \not \subset g^{+}(V)$. Then, for some $u \in U, g u \cap(Y-V) \neq \varnothing$, so $g_{\alpha} u \cap(Y-V) \neq \varnothing$ eventually, which is a contradiction.

LEMMA 3.2. If $F$ is an evenly continuous subset of $\left(Y^{m X}\right)_{0}$, then $\tau_{p}$ on $F$ is jointly continuous.

Proof. Let $\omega:\left(F, \tau_{p}\right) \times X \rightarrow Y$. Suppose that $(f, x) \in \omega^{-}(G)$, where $G$ is open in $Y$. Choose $y \in f x \cap G$. There are neighborhoods $U, W$ of $x, y$, respectively, such that $g \in F$ and $g x \cap W \neq \varnothing$ imply $U \subseteq g^{-}(G)$. Then $\{h: h \in F$ and $h x \cap W \neq \varnothing\} \times U$ is a neighborhood of $(f, x)$ which is contained in $\omega^{-}(G)$.

Now suppose that $(f, x) \in \omega^{+}(G)$, where $G$ is open in $Y$. There are neighborhoods $U, W$ of $x, f x$, respectively, such that $g \in F$ and $g x \subseteq W$ imply $U \subseteq g^{+}(G)$. Then $\{h: h \in F$ and $h x \subseteq W\} \times U$ is a neighborhood of $(f, x)$ which is contained in $\omega^{+}(G)$.

The following lemma generalizes an implicit lemma of Noble [8], stated explicitely as Lemma 1.4 in $[7$, p. 7]:

LeMma 3.3. Let $f \in \mathscr{C}(X \times Y, Z)$. If $X$ is compact and $Z$ is regular, then the set $F=\{f(x, \cdot): x \in X\}$ is evenly continuous.

Proof. Let $y \in Y$, let $K$ be a compact subset of $Z$ and let $V$ be an open neighborhood of $K$. Let $W$ be a closed neighborhood of $K$ which is contained in $V$. We construct a neighborhood $U$ of $y$ as follows: Since $f(\cdot, y)$ is continuous, $K_{1}=f(\cdot, y)^{-}(W)$ and $K_{2}=f(\cdot, y)^{+}(W)$ are closed in $X$, therefore compact. Thus the second projections $\mathrm{pr}_{2}: K_{1} \times$ $Y \rightarrow Y, \mathrm{pr}_{2}: K_{2} \times Y \rightarrow Y$ are closed, so that

$$
U_{1}=Y-\operatorname{pr}_{2}\left[\left(K_{1} \times Y\right)-f^{-}(V)\right], \quad U_{2}=Y-\operatorname{pr}_{2}\left[\left(K_{2} \times Y\right)-f^{+}(V)\right]
$$

are open in $Y$. Because $K_{1} \subseteq f(\cdot, y)^{-}(V), K_{2} \subseteq f(\cdot, y)^{+}(V)$, we have $K_{1} \times\{y\} \subseteq f^{-}(V), \quad K_{2} \times\{y\} \subseteq f^{+}(V)$. Hence $y \notin \operatorname{pr}_{2}\left[\left(K_{1} \times Y\right)-f^{-}(V)\right]$, $y \notin \operatorname{pr}_{2}\left[\left(K_{2} \times Y\right)-f^{+}(V)\right]$, that is, $y \in U_{1} \cap U_{2}=U$.

We show that the neighborhoods $U, W$ of $y, K$, respectively, satisfy the required implications: Let $g \in F$ be such that $g y \cap W \neq \varnothing$, so that $g=f(x, \cdot)$ for some $x \in K_{1}$. Let $u \in U$, so that $u \notin \operatorname{pr}_{2}\left[\left(K_{1} \times Y\right)-f^{-}(V)\right]$. 
Then $(x, y) \in f^{-}(V)$, that is, $g u \cap V \neq \varnothing$. Now let $g \in F$ be such that $g y \subseteq W$, so that $g=f(x, \cdot)$ for some $x \in X_{2}$. Let $u \in U$, so that $u \notin \operatorname{pr}_{2}\left[\left(K_{2} \times Y\right)-f^{+}(V)\right]$. Then $(x, u) \in f^{+}(V)$, that is, $g u \subseteq V$.

Let $X$ and $Y$ be topological spaces. The compact open topology $\tau_{c}$ on $Y^{m X}$ is defined to be the topology having as open subbase the sets of the forms $\{f: f(K) \subseteq U\},\{f: f x \cap U \neq \varnothing$ for all $x \in K\}$, where $K$ is a compact subset of $X$ and $U$ is open in $Y$. Obviously, $\tau_{c}$ is larger than $\tau_{p}$.

A subset $F$ of $Y^{m X}$ satisfies the condition $(G)$ if, for every $\tau_{c}$-closed subset $F_{0}$ of $F, \cap_{f \in F_{0}} f^{-}(U)$ and $\bigcap_{f \in F_{0}} f^{+}(U)$ are open in $X$ whenever $U$ is open in $Y$. The following two lemmas relate this condition to even continuity:

LEMMA 3.4. If $Y$ is regular, then every subset of $Y^{m X}$ satisfying the condition $(G)$ is evenly continuous.

Proof. Let $F$ be a subset of $Y^{m X}$ which satisfies the condition $(G)$. Let $x \in X$, let $K$ be a compact subset of $Y$ and let $V$ be an open neighborhood of $K$. Let $W$ be an open neighborhood of $K$ such that $K \subseteq W \subseteq \bar{W} \subseteq V$. Since $F_{1}=\{h: h \in F \quad$ and $h x \cap \bar{W} \neq \varnothing\}, \quad F_{2}=$ $\{h: h \in F$ and $h x \subseteq \bar{W}\}$ are $\tau_{c}$-closed in $F, U_{1}=\bigcap_{h \in F_{1}} h^{-}(V)$ and $U_{2}=$ $\bigcap_{h \in F_{2}} h^{+}(V)$ are open in $X$. Then $U=U_{1} \cap U_{2}$ is an open neighborhood of $x$.

Let $f \in F$ be such that $f x \cap W \neq \varnothing$. Then $f \in F_{1}$, so that $U \subseteq U_{1} \subseteq$ $f^{-}(V)$. Now let $f \in F$ be such that $f x \subseteq W$. Then $f \in F_{2}$, so that $U \subseteq U_{2} \subseteq f^{+}(V)$.

LEMMA 3.5. Every $\tau_{c}$-compact evenly continuous subset of $\left(Y^{m X}\right)_{0}$ satisfies the condition $(G)$.

Proof. Let $F$ be a $\tau_{c}$-compact evenly continuous subset of $\left(Y^{m X}\right)_{0}$. Since $F$ is $\tau_{p}$-compact, it suffices, by Corollary 10.6 of [7, p. 23], to show that $\tau_{p}$ on $F$ is jointly continuous. For this we apply Lemma 3.2.

Let $X$ be a topological space and let $Y=(Y, \mathscr{U})$ be a uniform space. A subset $F$ of $Y^{m X}$ is equicontinuous if, for $(x, U) \in X \times \mathcal{U}$, there exists a neighborhood $V$ of $x$ such that, for all $f \in F, f(V) \subseteq U[f x]$ and $f z \cap U[y] \neq \varnothing$ whenever $(z, y) \in V \times f x$. The following two lemmas relate equicontinuity to even continuity:

LEMMA 3.6. If $Y=(Y, \mathcal{U})$ is a uniform space, then every equicontinuous subset of $Y^{m X}$ is evenly continuous.

Proof. Let $F$ be an equicontinuous subset of $Y^{m x}$. Let $x \in X$, let $K$ be a compact subset of $Y$ and let $U$ be a symmetric member of $u$. There is a neighborhood $V$ of $x$ such that, for all $f \in F, f(V) \subseteq$ 
$U[f x]$ and $f x \subseteq U[f z]$ for all $z \in V$. Write $W=U[K]$. Let $f \in F$ be such that $f x \cap W \neq \varnothing$. If $z \in V$ then $f x \subseteq U[f z]$, so that $U[f z] \cap W \neq \varnothing$, therefore $V \subseteq f^{-}\left(U^{2}[K]\right)$. Now let $f \in F$ be such that $f x \subseteq W$. Then $f(V) \subseteq U[f x] \subseteq U^{2}[K]$, that is, $V \subseteq f^{+}\left(U^{2}[K]\right)$.

LEMMA 3.7. If $Y$ is a uniform space, then every evenly continuous pointwise bounded subset of $\left(Y^{m X}\right)_{0}$ is equicontinuous.

Proof. Let $F$ be an evenly continuous pointwise bounded subset of $\left(Y^{m X}\right)_{0}$. Let $\bar{F}$ denote the $\tau_{p}$-closure of $F$ in $\left(Y^{m X}\right)_{0}$. By the Lemmas $3.1,3.2, \tau_{p}$ on $\bar{F}$ is jointly continuous. Since $\left(Y^{m X}\right)_{0}$ is a Tychonoff set, by Lemma $2.1, \bar{F}$ is $\tau_{p}$-compact. Then, by the Lemma 8 of Smithson $[9, \mathrm{p}$. 258], $\bar{F}$ is equicontinuous.

4. Ascoli theorem. Let $X=(X, \tau)$ be a topological space. The $k$-extension of $\tau$ is the family $k(\tau)$ of all subsets $U$ of $X$ such that $U \cap K$ is open in $K$ for every compact subset $K$ of $X$. It is clear that $k(\tau)$ is a topology on $X$ which is larger than $\tau$. The topological space $k X=(X, k(\tau))$ is called the $k$-extension of $X$. A topological space $X$ is called a $k$-space if $k X=X$. For an arbitrary topological space $X, k k X=k X$, so $k X$ is a $k$-space. Familiar examples of $k$-spaces are the locally compact spaces and the spaces satisfying the first countability axiom.

Let $X$ and $Y$ be topological spaces. A function $f: X \rightarrow Y$ is called $k$-continuous if its restriction to each compact subset of $X$ is continuous. Henceforth, the set of all continuous ( $k$-continuous) functions on $X$ to $Y$ will be denoted $C(X, Y)\left(C_{k}(X, Y)\right)$. It can be shown that a topological space $X$ is a $k$-space if and only if $C_{k}(X, Y)=C(X, Y)$ for every topological space $Y\left[7\right.$, p. 9]. A topological space $X$ is a $k_{3}$-space if $C_{k}(X, Y)=C(X, Y)$ for every regular space $Y$. Thus a $k$-space is a $k_{3}$-space but not conversely. In fact, the product of uncountably many copies of the real line, which is not a $k$-space, is a $k_{3}$-space. We write $\mathscr{C}_{0}(X, Y)=\left(Y^{m X}\right)_{0} \cap \mathscr{C}(X, Y)$.

We note that if $Y$ is regular, then $\left(\mathscr{C}_{0}(X, Y), \tau_{c}\right)$ is a regular space for every topological space $X$.

In a regular space there was introduced in $[7, \mathrm{p} .11]$ the following equivalence relation $R: x R y$ if every open neighborhood of $x$ contains $y$. For a subset $F$ of such a space, $F^{*}$ denotes its $R$-saturation, that is, the smallest $R$-saturated set containing $F$.

THEOREM 4.1. Let $X, Y$ be topological spaces, let $T$ be a Tychonoff subset of $\left(Y^{m X}\right)_{0}$ and let $F \subseteq\left(T \cap \mathscr{C}(X, Y), \tau_{c}\right)$. If $Y$ is regular, the following conditions are sufficient for the compactness of $F$ : 
(a) $F^{*}$ is closed in $T \cap \mathscr{C}(X, Y)$.

(b) $F$ is pointwise bounded, and

(c) $F$ is evenly continuous.

If $X$ is a $k$-space and $Y$ is regular, then the conditions (a), (b) and (c) are necessary for the compactness of $F$.

Proof. Sufficiency. Let $\bar{F}$ denote the $\tau_{p}$-closure of $F$ in $T$. Since $T \subseteq\left(Y^{m X}\right)_{0}$, (c) implies, by Lemmas 3.1 and 3.2, that $\omega:\left(\bar{F}, \tau_{p}\right) \times X \rightarrow Y$ is continuous, and, in particular, that $\bar{F} \subseteq \mathscr{C}(X, Y)$. By Lemma 8.1 of [7, p. 18], $\tilde{\omega}:\left(\bar{F}, \tau_{p}\right) \rightarrow\left(\mathscr{C}(X, Y), \tau_{c}\right)$ is continuous. Since $T$ is a Tychonoff set, (b) implies, by Lemma 2.1 , that $\bar{F}$ is $\tau_{p}$-compact, so $\tilde{\omega}(\bar{F})=\bar{F}$ is a $\tau_{c}$-compact subset of $T \cap \mathscr{C}(X, Y)$. But (a) implies $F \subseteq \bar{F} \subseteq F^{*}$, so, by Theorem 4.1 (b) of [7, p. 11], $F$ is $\tau_{c}$-compact.

Necessity. By Theorem 4.1 (c) of [7, p. 11], $F^{*}$ is closed in $\left(T \cap \mathscr{C}(X, Y), \tau_{c}\right)$. It is clear that $F$ is pointwise bounded. Since $X$ is a $k$-space, by Theorem 9.4 of [7, p. 21], $\omega:\left(F, \tau_{c}\right) \times X \rightarrow Y$ is continuous. So by Lemma 3.3, $F=\{\omega(f, \cdot): f \in F\}$ is evenly continuous.

Corollary 1. Let $F \subseteq\left(\mathscr{C}_{0}(X, Y), \tau_{c}\right)$. If $Y$ is regular, the following conditions are sufficient for the compactness of $F$ :

(a) $F^{*}$ is closed in $\mathscr{C}_{0}(X, Y)$,

(b) $F$ is pointwise bounded, and

(c) $F$ is evenly continuous.

If $X$ is a $k$-space and $Y$ is regular, then the conditions (a), (b) and (c) are necessary for the compactness of $F$.

COROllary 2. Let $F \subseteq\left(C(X, Y), \tau_{c}\right)$. If $Y$. is regular, the following conditions are sufficient for the compactness of $F$ :

(a) $F^{*}$ is closed in $C(X, Y)$,

(b) $F$ is pointwise bounded, and

(c) $F$ is evenly continuous.

If $X$ is a $k$-space and $Y$ is regular, then the conditions (a), (b) and (c) are necessary for the compactness of $F$.

Corollary 3. If $Y$ is regular, a subset $F$ of $\left(C_{k}(X, Y), \tau_{c}\right)$ is compact if and only if

(a) $F^{*}$ is closed in $C_{k}(X, Y)$,

(b) $F$ is pointwise bounded, and

(c) $F$ is evenly continuous on compacta.

Proof. For the sufficiency, we note that $C_{k}(X, Y)=C(k X, Y)$ and apply the Lemma 3.4 of [7, p. 11]. For the necessity, we consider $F$ as a subset of $\left(C(k X, Y), \tau_{c}\right)$ and deduce from Corollary 2 the conditions (a), 
(b) and the even continuity of $F$. Then it is clear that $F$, considered as a subset of $\left(C_{k}(X, Y), \tau_{c}\right)$, is evenly continuous on compacta.

Corollary 4. ([1,p. 635]). Let $F \subseteq\left(C(X, Y), \tau_{c}\right)$. If $Y$ is regular, the following conditions are sufficient for the compactness of $F$ :

(a) $F$ is closed in $C(X, Y)$,

(b) $F$ is pointwise bounded, and

(c) $F$ is evenly continuous.

If $X$ is a $k_{3}$-space and $Y$ is regular, then the conditions (a), (b) and (c) are necessary for the compactness of $F$.

Proof. For the necessity, we note that, since $Y$ is regular, $C_{k}(X, Y)=C(X, Y)$; then we apply Corollary 3 and Lemma 3.4 of $[7, \mathrm{p}$. $11]$.

Remarks. (1) By Lemmas 3.4, 3.5, the Corollary 1 is equivalent to the Theorem 10.10 of [7, pp. 23-24], which contains the Ascoli theorem of Gale [2, p. 304] and the multifunction Ascoli theorem of Mancuso [6, p. 470].

(2) Let $Y$ be a uniform space. By Lemmas 3.6, 3.7 and Theorem 12.2 of $[7$, p. 28], the Corollary 1 in this context is equivalent to the Theorem 12.8 of $[7$, p. 31], which contains the multifunction Ascoli theorem of Smithson [9, p. 259].

\section{REFERENCES}

1. G. Fox and P. Morales, A non-Hausdorff Ascoli theorem for $k_{3}$-spaces, Proc. Amer. Math. Soc., 39 (1973), 633-636.

2. D. Gale, Compact sets of functions and function rings, Proc. Amer. Math. Soc., 1 (1950), 303-308.

3. J. Kelley, General Topology, New York 1965.

4. Y. F. Lin, Tychonoff's theorem for the space of multifunctions, Amer. Math. Monthly, 74 (1967), 399-400,

5. Y. F. Lin and D. A. Rose, Ascoli's theorem for spaces of multifunctions, Pacific J. Math., 34 (1970), 741-747.

6. V. J. Mancuso, An Ascoli theorem for multi-valued functions, J. Austral. Math. Soc., 12 (1971), 466-472.

7. P. Morales, Non-Hausdorff Ascoli theory, Dissertationes Math. 119 (1974), 1-37.

8. N. Noble, Ascoli theorems and the exponential map, Trans. Amer. Math. Soc., 143 (1969), 393-411.

9. R. Smithson, Uniform convergence for multifunctions, Pacific J. Math., 39 (1971), 253-259.

Received June 11, 1975.

Université de Montréal 




\section{Pacific Journal of Mathematics}

Vol. 64, No. 1

May, 1976

Walter Allegretto, Nonoscillation theory of elliptic equations of order $2 n \ldots \ldots \quad 1$

Bruce Allem Anderson, Sequencings and starters.................. 17

Friedrich-Wilhelm Bauer, A shape theory with singular homology .......... 25

John Kelly Beem, Characterizing Finsler spaces which are

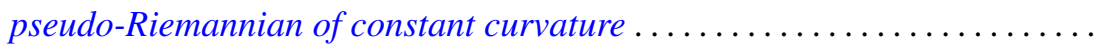

Dennis K. Burke and Ernest A. Michael, On certain point-countable

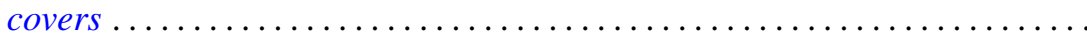

Robert Chen, A generalization of a theorem of Chacon ............... 93

Francis H. Clarke, On the inverse function theorem ................ 97

James Bryan Collier, The dual of a space with the Radon-Nikodým

property ....................................... 103

John E. Cruthirds, Infinite Galois theory for commutative rings ............ 107

Artatrana Dash, Joint essential spectra......................... 119

Robert M. DeVos, Subsequences and rearrangements of sequences in FK

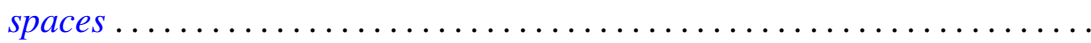

Geoffrey Fox and Pedro Morales, Non-Hausdorff multifunction generalization

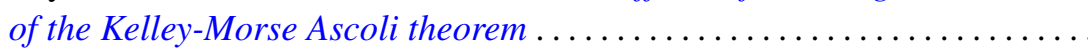

Richard Joseph Fleming, Jerome A. Goldstein and James E. Jamison, One

parameter groups of isometries on certain Banach spaces.............

Robert David Gulliver, II, Finiteness of the ramified set for branched

immersions of surfaces

Kenneth Hardy and István Juhász, Normality and the weak cb property ...... 167

C. A. Hayes, Derivation of the integrals of $L^{(q)}$-functions.

Frederic Timothy Howard, Roots of the Euler polynomials .

Robert Edward Jamison, II, Richard O'Brien and Peter Drummond Taylor, On

embedding a compact convex set into a locally convex topological vector space ....................................

Andrew Lelek, An example of a simple triod with surjective span smaller than span ...

Janet E. Mills, Certain congruences on orthodox semigroups

Donald J. Newman and A. R. Reddy, Rational approximation of $e^{-x}$ on the positive real axis.

John Robert Quine, Jr., Homotopies and intersection sequences ...

Nambury Sitarama Raju, Periodic Jacobi-Perron algorithms and fundamental units ....

Herbert Silverman, Convexity theorems for subclasses of univalent functions. . .

Charles Frederick Wells, Centralizers of transitive semigroup actions and endomorphisms of trees.........................

Volker Wrobel, Spectral approximation theorems in locally convex spaces ..................... 